\title{
Investigating Subjective Cognitive Complaints in Psychosis: Introducing the Brief Scale to Investigate Cognition in Schizophrenia (SSTICS-Brief)
}

\author{
Matteo Cella ${ }^{1,2}$, Michael Bodnar ${ }^{3}$, Martin Lepage ${ }^{4}$, Ashok Malla ${ }^{4}$, Ridha Joober ${ }^{4}$, Srividya Iyer ${ }^{4}$, Til \\ Wykes $^{1,2}$, Antonio Preti ${ }^{5}$ \\ 1 - Department of Psychology, Institute of Psychiatry, Psychology \& Neuroscience, King's College London, UK; \\ 2 - South London and Maudsley NHS Trust, London, UK. \\ 3 - The Royal Ottawa Institute of Mental Health Research, Ottawa, Ontario, Canada \\ 4 - Department of Psychiatry, McGill University \& Prevention and Early Intervention Program for Psychosis (PEPP), Douglas Mental \\ Health University Institute, Montreal, Canada \\ 5 - Center for Liaison Psychiatry and Psychosomatics, University Hospital, University of Cagliari, Cagliari, Italy.
}

Corresponding Author:

Dr. Matteo Cella

Department of Psychology,

Institute of Psychiatry, Psychology \& Neuroscience

King's College London,

De Crespigny Park,

SE5 8AF

London, UK

Telephone: (+44) 02078485001

Email: matteo.cella@kcl.ac.uk

This is an Accepted Manuscript of an article published by Taylor \& Francis in Cognitive Neuropsychiatry on February 6. 2020, available online: https://doi.org/10.1080/13546805.2020.1722084 


\section{Abstract}

Background: Cognitive difficulties are a core deficit for people with schizophrenia and are generally assessed with neuropsychological tests. However, self-report assessments are also useful in understanding difficulties from the service user's perspective. Self-report measures are only useful if they are acceptable and measure an important illness dimension. This study aims to introduce and test the shorter version of the Subjective Scale to Investigate Cognition in Schizophrenia (SSTICS) to improve this scale's acceptability and comprehensibility.

Methods: In consultation with service users and clinicians, we identified items from the original 21-item SSTICS that service users found difficult. These items were excluded, and the subsequent reduced scale was explored with Confirmatory Factor Analysis (CFA) in two independent samples of people with a diagnosis of schizophrenia or first-episode psychosis recruited in the UK and Canada. Convergent validity with symptoms and IQ was assessed and compared between the original and the reduced scale.

Results: Six-hundred and seven people with schizophrenia and first-episode psychosis took part in this study. Seven items were removed to produce the 14-item scale (i.e. SSTICS-B). This new scale had good reliability and the CFA confirmed a unidimensional structure. Convergent validity with symptoms and IQ were optimal indicating that the long and short version of the scale assess similar constructs.

Conclusions: By removing unclear items, the SSTICS-B has better acceptability than its longer form and could be administered in less time. The resulting measure is likely to be a valuable short self-assessment of cognitive complaints in people with schizophrenia.

Keywords: Cognition; Psychosis; Schizophrenia; Subjective; Assessment. 


\section{Introduction}

Cognitive difficulties are a core deficit for people with schizophrenia and exist prior to the onset of psychosis (Bora et al., 2014; Gur et al., 2014). The assessment of cognitive difficulties is performed using standardized tests which offer the opportunity to compare scores with population norms and are often referred to as objective assessments. The subjective experience of cognitive difficulties in people with schizophrenia has, however, received less attention. Interest in this area has been hampered by research showing not only that people with schizophrenia have poor insight about their symptoms but also poor insight into their cognitive difficulties (David, Bedford, Wiffen, \& Gilleen, 2012; Medalia \& Lim, 2004; Medalia \& Thysen, 2008). It has been observed that objective and subjective assessments of cognition often produce different results suggesting that subjectively reported difficulties may be a different aspect of the disorder (Harvey et al., 2001; Homayoun, Nadeau-Marcotte, Luck, \& Stip, 2011; Keefe, Poe, Walker, Kang, \& Harvey, 2006; Stip, Caron, Renaud, Pampoulova, \& Lecomte, 2003).

Reporting cognitive problems to a clinician can serve as a useful starting point for considering interventions targeting cognition and providing a rationale that is congruent with the client's perceptions. A self-assessment of cognition can also be compared to a concurrent neuropsychological assessment to assess potential differences, with large discrepancies having potential implications for therapy engagement, realistic goal setting and self-esteem.

For these reasons, self-assessment of cognitive difficulties should be included more often as part of standard assessments. The Subjective Scale to Investigate Cognition in Schizophrenia (SSTICS) is one of the most used self-assessment tools for people with schizophrenia (Potvin, Aubin, \& Stip, 2017; Stip et al., 2003). The SSTICS is a 21 -item scale rating the frequency of common cognitive problems. Its psychometric properties are satisfactory, and many studies have used this measure to date (e.g. Lecardeur et al., 2009; Potvin et al., 2005; Sellwood et al., 2013). Studies investigating its factorial structure have suggested that it is both unidimensional and multidimensional with Potvin et al (Potvin et al., 2017) suggesting this scale has five factors corresponding to different neuropsychological domains. To date, however, the unidimensional structure of the instrument is the one supported by the best quality evidence and all but one study (Potvin et al., 2017) used this scale as unidimensional.

The SSTICS can be used as a screening measure and be completed by service users independently. However, item complexity may limit self-administration which can affect item interpretation. This study initially aims to examine the SSTICS items to discover which are difficult for service users to understand. We will then remove any overly complex items and test the resulting briefer scale for its psychometric proprieties and convergent validity to measures of symptoms and intelligence quotient (IQ) using data from two independent samples. A shorter measure producing similar results to its longer version can offer significant advantages including a shorter completion time and a higher likelihood of service users being able to complete the measure independently. 


\section{Methods}

\subsection{Identifying difficult to understand items}

\section{Participants}

Service users with a diagnosis of schizophrenia and clinicians were recruited via community services at the South London and Maudsley NHS Foundation Trust. Service users were referred by care coordinators as having cognitive difficulties. Clinicians working in psychosis services with familiarity with the SSTICS and cognitive difficulties in psychosis were approached to take part.

\section{Procedure}

Fifteen service users (12 males; average age 36.6) were asked to identify SSTICS items which may be unclear or ambiguous by highlighting all items that they considered difficult to understand. They were asked to comment on the comprehensibility of the item, clarity of the question and request from the instruction. Five clinicians (e.g. clinical and assistant psychologists) who use this measure regularly were also asked to indicate, separately, the items that service users find difficult to complete on their own and which require further explanation. All participants were asked for feedback and comments on the scale individually and their comments recorded verbatim. Feedback was collated and reviewed by MC, MB and two independent service users' advisors. Items that received the most suggestions for reconsideration from our service users and clinicians (i.e. five or more service users and at least two clinicians recommended) were put forward for exclusion.

\subsection{Testing the SSTICS-Brief (SSTICS-B)}

\section{Participants}

Baseline data from two independent research studies with similar inclusion and exclusion criteria were used for the study. One study was based in the UK and the other in Canada. In the UK, individuals who received care from specialized mental health care services for people with psychosis at the South London and Maudsley NHS Foundation Trust were recruited. This includes people with schizophrenia, schizoaffective disorders who received care from community mental health teams but are not under the care of early intervention psychosis teams (i.e. excludes people with first episode psychosis).

Service users from Canada were recruited via the Prevention and Early Intervention Program for Psychoses (PEPP-Montreal), a specialized early intervention service at the Douglas Mental Health University Institute in Montreal. This early intervention program provides services to individuals aged 14 to 35 years 
from a defined local catchment area who are experiencing either a non-affective or affective first-episode of psychosis (FEP) and have not taken antipsychotic medications for more than $\mathbf{3 0}$ days.

Services users at both sites were out-patients. Exclusion criteria for both sites were presence of learning disability, organic brain damage, pervasive developmental disorder, or epilepsy.

\section{Measures}

\section{Self-assessed Cognitive difficulties}

The SSTICS is a 21-item measure focusing on difficulties associated with core cognitive domains including memory, attention, executive functions and praxia. Each item is rated on a five-point Likert scale ranging from 'never' to 'very often' with higher scores suggesting more cognitive problems. A problem score represents those difficulties that service users are likely to mention in a consultation and which are those for which they would seek treatment. The total problem score is the number of items endorsed at the 'very often' or 'often' level. The measure has good internal consistency ( $\alpha=0.86)$ and test-retest reliability ( $r=0.8$ ) (Stip et al., 2003).

\section{Symptoms}

Symptom severity was measured using the Positive and Negative Syndrome Scale (PANSS) (Kay, Fiszbein, \& Opler, 1987) assessing symptoms in the past week. For this study, we considered the three symptom factors of the PANSS: i) positive symptoms, ii) negative symptoms and iii) general symptoms. Data were collected by trained interviewers on both sites and used to estimated convergence validity (i.e. positive correlation between symptoms severity and self-reported cognitive difficulties).

\section{IQ estimate (Canada study only)}

IQ was estimated using the Wechsler Abbreviated Adult Intelligence Scale - Third Edition (WAIS-III) (Wechsler, 1997) or the Wechsler Abbreviated Scale of Intelligence - First Edition (WASI-I) (Wechsler, 1999).

\section{Procedures}

Participants completed the measures during an initial assessment as part of different research and clinical protocols. All participants provided written informed consent. Research protocols were approved by NHS Research Ethics in the UK (08/H0807/26 and 13/LO/1791) and the Research Ethics Board of the Douglas Mental Health University Institute and the McGill University Faculty of Medicine (IUSMD 08/26) in Canada.

\subsection{Statistics}


Descriptive statistics were reported for both the UK and Canadian samples. Reliability of the full and the short SSTICS was measured using Cronbach's alpha and McDonald's omega, as estimated by the confirmatory factor analysis model (McDonald, 1985). Values of the McDonald's omega around 0.9 are considered appropriate monofactorial measures.

The factor structure for the SSTICS and the SSTICS-B were tested by confirmatory factorial analysis (CFA), computed using the lavaan package (Rosseel, 2012) for $R$ (Team, 2017) independently in each of the two samples. We tested a unidimensional model for both the SSTICS and SSTICS-B. A factor loading of 0.32 ( $10 \%$ of the variance) was the minimum requirement for an item to be retained (Comrey \& Lee, 1992). We used the Mardia's test to assess violation of multivariate normality in the data. When normality was not confirmed, we treated items as ordinal variables and used polychoric correlations (Joreskog, 1994).The diagonally weighted least squares (DWLS) estimator was used to assess the model's fit. The DWLS estimator is known to have a good control of Type I error rates with sample sizes as small as 200 (Flora \& Curran, 2004). Fit estimation was estimated using a number of parameters including a non-significant chi square, the comparative fit index (CFI 0.9 or higher), the root mean square error of approximation (RMSEA 0.08 or lower), and the standardized root mean square residual (SRMR 0.09 or lower) were considered indicators of acceptable fit (Hu \& Bentler, 1999).

Convergent validity was tested by correlating SSTICS or SSTICS-B problem scores with symptom severity (PANSS total and factors) in both samples independently, and with estimated verbal and performance Intelligence Quotient (IQ) in the Canadian sample. We use the Steiger test to compare the strength of correlation coefficients between the SSTICS and the SSTICS-B by using the "cocor" package running in R (Diedenhofen \& Musch, 2015).

Lastly, we assessed measurement invariance of the unidimensional model across the samples for the STICSS and SSTICS-B the using multi-group CFA according to Byrne and van de Vijver (Byrne \& van de Vijver, 2010) and using the R semTools package (Jorgensen, 2018). Configural, metric and scalar invariance was tested, starting from the identification of a well-fitting baseline model and then establishing successive equivalence constraints in the model parameters across the groups. Configural invariance was used to assess if the same CFA model is valid in each group. Metric invariance was used to assess if the factorial loadings was equivalent across the groups. Scalar invariance was used to assess if the item intercepts and the factor loadings are equally constrained across groups. Since the chi-square test is very sensitive to sample size (Bollen \& Long, 1992), models were assessed based on changes in CFI and RMSEA (delta-CFI and delta-RMSEA). To assess measurement invariance the following criteria were used: the fit of the model, and the conventionally agreed threshold of 0.01 for delta-CFI and 0.02 for delta-RMSEA (Chen, 2007; Cheung \& Rensvold, 2002). 


\section{Results}

\section{Item removal}

Seven items were removed to produce the SSTICS-B. Items put forward for reconsideration were too long and wordy or suggested examples that were not relevant or applicable to the person (e.g. "do you have difficulties remembering how to get to the hospital" to a community patient). Reasons for item exclusion were: irrelevance, poor formulation, difficult to keep all the information in mind and unclear question. The items suggested for exclusion by this process were items 4, 6, 9, 14, 18, 19 and 21.

\section{Testing SSTICS-B}

The detailed characteristics of the samples are shown in Table 1. The samples had a similar sample size and gender distribution but were of different ages with service users from Canada being, on average, over 10 years younger. Duration of illness and diagnosis were different between the two samples with all participants from the UK having a diagnosis of schizophrenia or schizoaffective disorder and illness duration over 5 years and all participants from Canada having a diagnosis of first episode psychosis and less the 5 years of illness duration. STICCS and STICCS-B problem scores were higher in the UK than the Canadian sample (Welch's t-test SSTICS Problem-score, $\mathrm{t}=-7.01$, d.f. $=491.1, \mathrm{p}<0.0001$; SSTICS-B Problem-score $\mathrm{t}=$ 6.65 , d.f. $=505.9, p<0.0001)$. Reliability measured using Cronbach's alpha was similar in the two samples for the full scale and for the SSTICS-B (SSTICS UK=0.91, Canada=0.90; SSTICS-B UK=0.89, Canada=0.88)

---- Table 1 about here ----

\subsection{Confirmatory factor analysis}

UK - The fit of the unidimensional model was good for both the SSTICS and the SSTICS-B (Table 2 and 3). Only 5 out of 21 items of the original SSTICS had a standardized factor loading lower than 0.5 , and 4 of them were items suggested for reconsideration by service users and clinicians.

Canada - The fit of the unidimensional model was also good for both versions of the scale (Table 2 and 3). Six out of 21 items of the original SSTICS had a standardized factor loading lower than 0.5 , and 4 of them were items suggested for reconsideration by service users and clinicians. In both samples, the McDonald omega was slightly lower for the SSTICS-B compared to the full version, but still within the acceptable range.

\subsection{Convergent validity}


In the UK sample, both the SSTICS and STICSS-B Problem-scores correlated positively with the PANSS General score $(r=0.251, p<0.0001 ; r=0.249, p<0.0001$ respectively) and did not differ (Steiger's test: $z=0.12, p=0.90)$. But neither score correlated with the Positive $(r=0.096, p=0.116 ; r=0.084, p=$ $0.171)$ or Negative $(r=-0.03, p=0.628 ; r=-0.038, p=0.538)$ scores.

In the Canadian sample, the pattern was different. The SSTICS Problem-score and the SSTICS-B Problem-score were both correlated with the PANSS Positive score $(r=0.182, p=0.004$ and $r=0.139, p=$ 0.030 , respectively) but not with the PANSS General score $(r=0.065, p=0.31$ and $r=0.050, p=0.44)$ or the PANSS Negative score $(r=0.001, p=0.98$ and $r=-0.018, p=0.77)$. The PANSS Positive score correlations was significantly higher for the SSTICS than SSTICS-B (Steiger's test: $z=3.14, p=0.0017$ ).

In the Canadian sample, the SSTICS and STICSS-B Problem-score correlated negatively with verbal ( $r$ $=-0.141, p=0.008 ; r=-0.112, p=0.034)$ and performance $\mathrm{IQ}(r=-0.152, p=0.004 ; r=-0.135, p=0.011)$ and the correlation coefficients differed for the verbal (Steiger's test verbal IQ, $z=2.64, p=0.008$ ) but not on the performance IQ (performance IQ, $\mathrm{z}=1.55, \mathrm{p}=0.12$ ).

\subsection{Measurement invariance}

The measurement invariance fit was good for all indicators except for the chi-square, as often with a large (> 500) sample size. However, there was a degrading of the fit with increasing constraints of the parameters (see Table 4). The degrading was more pronounced from the metric model to the scalar model for both SSTICS and the SSTICS-B. Overall, the threshold for acceptance of measurement invariance was satisfactorily retained for the RMSEA and the CFI.

---- Table 4 about here ----

\section{Discussion}

In this study, we introduced and tested a brief version of the SSTICS in two independent samples of people with psychosis. The UK sample included individuals with longer illness duration while the Canadian included individuals with first-episode psychosis. These two samples differed in illness length and symptom severity with the UK sample showing higher levels of general symptoms while the Canadian sample showed higher positive symptoms.

In the first phase of this study, we asked service users and clinicians to identify items on the SSTICS that were hard to understand. They identified seven items to be put forward for removal and four of those were also identified by our factor analysis. After removing the seven items, the SSTICS-B was shown to have good psychometric properties similar to the full-length scale in two independent samples from different countries considering service users at different illness stages. The CFA for the SSTICS-B showed good fit for the unidimensional model, similar to the original scale. In the UK sample, which included patients with a 
longer illness duration, the SSTICS-B showed a positive but modest correlation with the PANSS General factor score. This suggests that in non-acute patients, the SSTICS-B may have a stronger association with aspects related to poor illness awareness and perhaps lack of self-esteem. The PANSS general factor includes items on poor insight, depression and anxiety which may influence awareness and self-esteem. Indeed, previous research has suggested that self-esteem is important in predicting levels of perceived cognitive problems in people with psychosis (Cella, Swan, Medin, Reeder, \& Wykes, 2014). If not used alongside neuropsychological tests, it may be appropriate to consider using a self-assessment measure of cognition together with an assessment of mood or self-esteem as individuals with low self-esteem are more likely to overreport problems.

In the Canadian sample, which included service users with a first episode of psychosis, the SSTICS-B correlated with positive symptoms, suggesting that in the acute phase of the illness, delusions and hallucinations may have a more pronounced influence on the perception of cognitive difficulties. This finding may also reflect the impact that positive symptoms may have on cognition (Cuesta et al., 2012; Jenkins, Bodapati, Sharma, \& Rosen, 2018; Loberg, Jorgensen, Kroken, \& Johnsen, 2015) with this point further supported by the negative correlation between the SSTICS-B and verbal and performance IQ. However, the strength of these associations was very small, and this may be a further indication of the limited overlap between performance-based and subjective measures of cognitive difficulties.

We also evaluated measurement invariance for the unidimensional model of both the long and the brief version of the SSTICS. There was indication of invariance for two out of the three criteria we used (i.e. the RMSEA and CFI indicators) suggesting that the total scores on the SSTICS-B can be considered comparable in the two samples. This is reassuring as the two samples differed by country of origin, language but also disorder stage (chronic versus first-episode). This suggests that the SSTICS-B is robust in both translations and can assess self-reported cognitive difficulties at different illness stages. However, for some SSTICS-B items there was indication of different loading; for example, item 11 has a higher loading on the latent construct in the UK compared to the Canadian sample, and the reverse was found for item 20. While these differences suggest potential slight variations in item interpretation in the two versions of the scale these were not large and therefore, we opted to retain these items.

This study has limitations. The extent and the scale of the service users and clinicians' feedback could have been more comprehensive, conducted at both sites and using a more rigorous method. One example of this is the COSMIN framework (Terwee et al., 2018) for selecting valuable and useful outcome measures. A second limitation is the lack of a more comprehensive cognitive assessments alongside the SSTICS. While this would be desirable in the context of this study, to assess the relationship between objective and subjective cognitive difficulties across the two samples and different versions of the scale, this would not have contributed to evaluate the SSTICS-B validity. There is increasing evidence suggesting that subjective and objective ratings of cognitive difficulties tend to correlate very little, if at all, in people 
with psychosis (Sellwood et al., 2013). This, implicitly, begs the question of how to validate subjective experiences and if subjective experiences should be considered at face value. However, this is a wider issue and it is beyond the scope of this paper to resolve. A further limitation is the fact that the SSTICS-B was embedded in the SSTICS and therefore it was not evaluated as a stand-alone measure. We also did not evaluate comprehensiveness which is a key component of content validity.

The SSTICS was originally designed for people with long-standing mental health difficulties living in supported settings. Current models of care for people with schizophrenia are oriented more towards community services, early intervention and prevention. The SSTICS-B, with removed items that were not applicable or difficult to read, may be more appropriate for community service users; it will reduce administration time and improve the likelihood to be completed by service users independently. The use of this scale may be particularly useful for screening and in the context of interventions tackling cognition such as cognitive remediation, and it may be particularly indicated for remediation programs tackling awareness of cognitive problems alongside cognitive difficulties (Reeder et al., 2017). The SSTICS brief version may also increase the opportunity to use self-assessment measures of cognition in both research and clinics and thus to routinely consider service users' views of their personal cognitive difficulties.

\section{Acknowledgment}

This work was supported in part supported by the National Institute for Health Research (NIHR) Mental Health Biomedical Research Centre at the South London and Maudsley NHS Foundation Trust and King's College London. This work was also supported by the Canadian Institutes of Health Research; the Fonds de Recherche du Quebec - Santé (FRSQ) and Sackler Foundation. 


\section{SSTICS-Brief}

\section{Subjective Scale to Investigate Cognition in Schizophrenia Brief version}

Reference: Cella M, Bodnar M, Lepage M, Malla A, Joober R, Iyer S, Wykes T, Preti A. (2020). Investigating subjective cognitive complaints in psychosis: Introducing the brief scale to Investigate cognition in schizophrenia (SSTICSBrief). Cognitive Neuropsychiatry, 25(3):190-200. doi: 10.1080/13546805.2020.1722084.

\section{INSTRUCTIONS}

On the sheet before you there is a series of questions on thinking skills common problems (e.g. memory and concentration) that you may have experienced while going about your everyday activities. We would like to find out how often you have noted such problems recently. If you find that any of the descriptions below corresponds to a problem you had, please select how often it has affected your life in the last month.

Use the scale below to rate each statement. Circle the number that best corresponds to your experience:

- 4 very often

- 3 often

- 2 sometimes

- 1 rarely

- 0 never

1. Have you noticed any difficulty remembering things?

- 4 very often

- 3 often

- 2 sometimes

- 1 rarely

- 0 never 
2. Do you have difficulty remembering information that is freshly received and that must be used immediately such as a telephone number, an address, a room number, a bus route number or somebody's name?

- 4 very often

- 3 often

- 2 sometimes

- 1 rarely

- 0 never

3. Do you have difficulty memorising things, such as a grocery list or a list of names?

- 4 very often

- 3 often

- 2 sometimes

- 1 rarely

- 0 never

4. Do you ever forget things, such as a date with a friend or a doctor's appointment?

- 4 very often

- 3 often

- 2 sometimes

- 1 rarely

- 0 never 
5. Do you have difficulty remembering information that you read in the newspapers or hear on TV?

- 4 very often

- 3 often

- 2 sometimes

- 1 rarely

- 0 never

6. Do you have difficulty doing household chores or repairs? For example, do you ever forget how to cook things or what ingredients go into a recipe?

- 4 very often

- 3 often

- 2 sometimes

- 1 rarely

- 0 never

7. Do you have difficulty remembering the names of well-known people, such as the Prime Minister?

- 4 very often

- 3 often

- 2 sometimes

- 1 rarely

- 0 never 
8. Do you have difficulty remembering national capitals, important dates in history, names of countries on other continents, or major scientific discoveries?

- 4 very often

- 3 often

- 2 sometimes

- 1 rarely

- 0 never

9. Are you absent-minded or up in the clouds? For example, you lose your train of thought in a conversation because you are distracted, or you have a hard time focussing on what you are reading.

- 4 very often

- 3 often

- 2 sometimes

- 1 rarely

- 0 never

10.Do you have difficulty being on the alert or reacting to unexpected situations? For example, a fire alarm or a car that rushes by suddenly as you are crossing the street.

- 4 very often

- 3 often

- 2 sometimes

- 1 rarely

- 0 never 
11. Are you unable to do two things at once? For example, memorise an address while making coffee, or count the money in your wallet while the talking to a shopkeeper.

- 4 very often

- 3 often

- 2 sometimes

- 1 rarely

- 0 never

12. Do you have trouble focussing your attention on the same thing for more than 20 minutes? For example, a TV programme or a book reading or during a lesson in a classroom.

- 4 very often

- 3 often

- 2 sometimes

- 1 rarely

- 0 never

13.Do you have difficulty planning out your activities as easily as you used to? For example, charting an itinerary for getting someplace, making a budget for the month, preparing meals or making time for laundry.

- 4 very often

- 3 often

- 2 sometimes

- 1 rarely

- 0 never

14. Do you have difficulty finding your words, forming sentences, understanding the meaning of words, pronouncing words or naming objects?

- 4 very often

- 3 often

- 2 sometimes

- 1 rarely

- 0 never 


\section{References}

Bollen, K. A., \& Long, J. S. (1992). Tests for Structural Equation Models - Introduction. Sociological Methods \& Research, 21(2), 123-131. doi:Doi 10.1177/0049124192021002001

Bora, E., Lin, A., Wood, S. J., Yung, A. R., McGorry, P. D., \& Pantelis, C. (2014). Cognitive deficits in youth with familial and clinical high risk to psychosis: a systematic review and metaanalysis. Acta Psychiatr Scand, 130(1), 1-15. doi:10.1111/acps.12261

Byrne, B. M., \& van de Vijver, F. J. R. (2010). Testing for Measurement and Structural Equivalence in Large-Scale Cross-Cultural Studies: Addressing the Issue of Nonequivalence. International Journal of Testing, 10(2), 107-132. doi:10.1080/15305051003637306

Cella, M., Swan, S., Medin, E., Reeder, C., \& Wykes, T. (2014). Metacognitive awareness of cognitive problems in schizophrenia: exploring the role of symptoms and self-esteem. Psychol Med, 44(3), 469-476. doi:10.1017/S0033291713001189

Chen, F. F. (2007). Sensitivity of goodness of fit indexes to lack of measurement invariance. Structural Equation Modeling-a Multidisciplinary Journal, 14(3), 464-504. doi:Doi 10.1080/10705510701301834

Cheung, G. W., \& Rensvold, R. B. (2002). Evaluating goodness-of-fit indexes for testing measurement invariance. Structural Equation Modeling-a Multidisciplinary Journal, 9(2), 233-255. doi:Doi 10.1207/S15328007sem0902_5

Comrey, A. L., \& Lee, H. B. (1992). A first course in factor analysis (2nd ed.). Hillsdale, N.J.: L. Erlbaum Associates.

Cuesta, M. J., de Jalon, E. G., Campos, M. S., Ibanez, B., Sanchez-Torres, A. M., \& Peralta, V. (2012). Duration of untreated negative and positive symptoms of psychosis and cognitive impairment in first episode psychosis. Schizophr Res, 141(2-3), 222-227. doi:10.1016/j.schres.2012.08.019

David, A. S., Bedford, N., Wiffen, B., \& Gilleen, J. (2012). Failures of metacognition and lack of insight in neuropsychiatric disorders. Philos Trans R Soc Lond B Biol Sci, 367(1594), 1379-1390. doi:10.1098/rstb.2012.0002

Diedenhofen, B., \& Musch, J. (2015). cocor: A Comprehensive Solution for the Statistical Comparison of Correlations. PLoS One, 10(4). doi:ARTN e0121945

10.1371/journal.pone.0121945

Flora, D. B., \& Curran, P. J. (2004). An empirical evaluation of alternative methods of estimation for confirmatory factor analysis with ordinal data. Psychological Methods, 9(4), 466-491. doi:10.1037/1082-989x.9.4.466

Gur, R. C., Calkins, M. E., Satterthwaite, T. D., Ruparel, K., Bilker, W. B., Moore, T. M., ... Gur, R. E. (2014). Neurocognitive growth charting in psychosis spectrum youths. JAMA Psychiatry, 71(4), 366-374. doi:10.1001/jamapsychiatry.2013.4190

Harvey, P. D., Serper, M. R., White, L., Parrella, M. J., McGurk, S. R., Moriarty, P. J., . . Davis, K. L. (2001). The convergence of neuropsychological testing and clinical ratings of cognitive impairment in patients with schizophrenia. Compr Psychiatry, 42(4), 306-313. doi:10.1053/comp.2001.24587a

Homayoun, S., Nadeau-Marcotte, F., Luck, D., \& Stip, E. (2011). Subjective and Objective Cognitive Dysfunction in Schizophrenia - is there a Link? Front Psychol, 2, 148. doi:10.3389/fpsyg.2011.00148 
Hu, L. T., \& Bentler, P. M. (1999). Cutoff Criteria for Fit Indexes in Covariance Structure Analysis: Conventional Criteria Versus New Alternatives. Structural Equation Modelinga Multidisciplinary Journal, 6(1), 1-55. doi:10.1080/10705519909540118

Jenkins, L. M., Bodapati, A. S., Sharma, R. P., \& Rosen, C. (2018). Working memory predicts presence of auditory verbal hallucinations in schizophrenia and bipolar disorder with $\begin{array}{lllll}\text { psychosis. } J \quad \text { Exp Neuropsychol, } & 40(1),\end{array}$ doi:10.1080/13803395.2017.1321106

Joreskog, K. G. (1994). On the Estimation of Polychoric Correlations and Their Asymptotic Covariance-Matrix. Psychometrika, 59(3), 381-389. doi:Doi 10.1007/Bf02296131

Jorgensen, H. A. (2018). semTools: Useful tools for structural equation modeling. Retrieved from https://CRAN.R-project.org/package=semTools

Kay, S. R., Fiszbein, A., \& Opler, L. A. (1987). The positive and negative syndrome scale (PANSS) for schizophrenia. Schizophr Bull, 13(2), 261-276.

Keefe, R. S. E., Poe, M., Walker, T. M., Kang, J. W., \& Harvey, P. D. (2006). The schizophrenia cognition rating scale: An interview-based assessment and its relationship to cognition, real-world functioning, and functional capacity. American Journal of Psychiatry, 163(3), 426-432.

Lecardeur, L., Stip, E., Giguere, M., Blouin, G., Rodriguez, J. P., \& Champagne-Lavau, M. (2009). Effects of cognitive remediation therapies on psychotic symptoms and cognitive complaints in patients with schizophrenia and related disorders: a randomized study. Schizophr Res, 111(1-3), 153-158. doi:10.1016/j.schres.2009.03.029

Loberg, E. M., Jorgensen, H. A., Kroken, R. A., \& Johnsen, E. (2015). Auditory verbal hallucinations reflect stable auditory attention deficits: a prospective study. Cogn Neuropsychiatry, 20(1), 81-94. doi:10.1080/13546805.2014.977857

McDonald, R. P. (1985). Factor analysis and related methods. Hillsdale, N.J.: Lawrence Erlbaum Associates.

Medalia, A., \& Lim, R. (2004). Treatment of cognitive dysfunction in psychiatric disorders. J Psychiatr Pract, 10(1), 17-25.

Medalia, A., \& Thysen, J. (2008). Insight into neurocognitive dysfunction in schizophrenia. Schizophr Bull, 34(6), 1221-1230.

Potvin, S., Aubin, G., \& Stip, E. (2017). [Subjective cognition in schizophrenia]. Encephale, 43(1), 15-20. doi:10.1016/j.encep.2016.01.002

Potvin, S., Briand, C., Prouteau, A., Bouchard, R. H., Lipp, O., Lalonde, P., . . Stip, E. (2005). CANTAB explicit memory is less impaired in addicted schizophrenia patients. Brain Cogn, 59(1), 38-42. doi:10.1016/j.bandc.2005.04.002

Reeder, C., Huddy, V., Cella, M., Taylor, R., Greenwood, K., Landau, S., \& Wykes, T. (2017). A new generation computerised metacognitive cognitive remediation programme for schizophrenia (CIRCuiTS): a randomised controlled trial. Psychol Med, 47(15), 27202730. doi:10.1017/S0033291717001234

Sellwood, W., Morrison, A. P., Beck, R., Heffernan, S., Law, H., \& Bentall, R. P. (2013). Subjective cognitive complaints in schizophrenia: relation to antipsychotic medication dose, actual cognitive performance, insight and symptoms. PLoS One, 8(12), e83774. doi:10.1371/journal.pone.0083774

Stip, E., Caron, J., Renaud, S., Pampoulova, T., \& Lecomte, Y. (2003). Exploring cognitive complaints in schizophrenia: the subjective scale to investigate cognition in schizophrenia. Compr Psychiatry, 44(4), 331-340. doi:10.1016/S0010-440X(03)000865

Team, R. C. (2017). R: A language and environment for statistical computing: R Foundation for Statistical Computing, Vienna, Austria. Retrieved from http://www.Rproject.org/ 
Terwee, C. B., Prinsen, C. A. C., Chiarotto, A., Westerman, M. J., Patrick, D. L., Alonso, J., . . Mokkink, L. B. (2018). COSMIN methodology for evaluating the content validity of patient-reported outcome measures: a Delphi study. Qual Life Res, 27(5), 1159-1170. doi:10.1007/s11136-018-1829-0

Wechsler, D. (1997). Wecshler Adult Intelligence Scale - 3rd Edition. San Antonio, TX: The Psychological Corporation.

Wechsler, D. (1999). Wechsler Abbreviated Scale of Intelligence. San Antonio, TX: The Psychological Corporation. 